\title{
Improvement of livebearer fish reproductive performance using Oocyte Developer (OODEV)
}

\author{
Andri Iskandar ${ }^{1}$, Muhammad A. Rafiuddin ${ }^{1}$, and Agus $O$. Sudrajat ${ }^{2}$ \\ ${ }^{1}$ Production Technology and Management of Aquaculture, College of Vocational studies, IPB \\ University, Indonesia \\ ${ }^{2}$ Department of Aquaculture, Faculty of Fisheries and Marine Science, IPB University, Indonesia
}

\begin{abstract}
Livebearer fish have a unique reproduction by internal fertilization without supplied nutrition from parents. However, increasing the frequency of spawning remains unknown. Therefore, the study aimed to improve reproductive performance by expanding the spawning frequency in three livebearer fish species: guppy, black molly, and platy. Four sets of 10 fish ( 6 females and 4 males) aged 80 days after birth were maintained in controlled media at $25-28{ }^{\circ} \mathrm{C}$ in the separated tank sized $20 \times 20 \times 15 \mathrm{~cm}$. All the fish were fed commercially and mixed with oocyte developer (OODEV) for 14 days at satiation. By using the same fish, spawning and rematuration were observed for 30 and 45 days respectively. The reproductive performance was evaluated for 45 days. The OODEVtreated broodstock showed birthing the larvae in all tanks on the $7^{\text {th }}$ day after treatment, while control fish displayed on the 14th day. The percentage of pregnant broodstock was $66.6-100 \%$ in each tank, indicating the OODEV-supplemented diet can be used to increase oocyte growth in the gonad. In the rematuration experiment, the fish spawned a 3 -fold increase in frequencies of spawning. These data suggested that OODEV can stimulate oocyte growth and increase the birthing frequency in livebearers.
\end{abstract}

\section{Introduction}

Unlike mammals, guppy, swordtail, and black molly belong to the livebearer. These fish are models to study for viviparous fish, which has a unique characterization of reproduction. Due to the absence of gonoduct, the connection of the ovary to the exterior is formed by its posterior region, deprived germinal cells, gestation occurs in the ovary without transferring nutrition from the parent. In viviparous, mammals transfer the nutrient to the offspring by blood plasma into the fetus via a placenta and umbilical cords [1]. The nutrition of the embryos during gestation establishes essential changes related to the difference in the site of pregnancy to the maternal ovary. This condition involves new relationships during embryonic development, as the possible acquisition of nutrients.

Consequently, the nutrition revealed the potential disposition of nutrients stored in the egg during the oogenesis and obtained further from the maternal tissues during gestation [2], regarding the sperm activity formed by spermatophore (called micro pocket) still no information related to the period kept in gonad in livebearer including guppy, swordtails, and 
black molly. The structure of active sperm as in micro pockets was stated from Kobayashi and Iwamatsu [3].

It is well known that oocyte growth and maturation have occurred through the brainpituitary-gonad (BPG axis) [4]. However, the BPG axis is a significant problem in teleost when the process is not achieved $[5,6]$. The issue through these pathways, fish can spawn only once a year, fish cannot be released the mature eggs naturally, and fish is unable to complete the oocyte growth. According to this thought, we try the oocyte developer (OODEV), a commercial product from IPB University, and have achieved an award from the Innovation business center and Governor of West Java. The OODEV contains gonadotrophin from equine (common name pregnant mare serum gonadotrophin, PMSG) and domperidone (a drug with dopamine antagonist activity). In this premix of OODEV, the PMSG may be in the brain and pituitary, affecting the release of the FSH endogenously in the pituitary, which has to involve the gonad to produce estradiol. Estradiol production is necessary to synthesize vitellogenin in the liver through the blood vessel. Meanwhile, domperidone is a drug that can block the endogenous dopamine in the brain [7]. By using OODEV containing the combination of PMSG and domperidone, we thought that the production of livebearer will increase with increasing the frequencies of spawning.

The livebearer has a short life for entering the market, and it is beneficial for the farmer as an additional income. Up to date, the production of livebearer based-local farmers in Bogor is needed around 5000 in a week. However, the local farmer can produce only around 2500 in a week, especially in the dry season. They depend on nature and try to be fed by silkworm or tubifex twice a day. However, the effort was not significantly changing the situation. Therefore, one effort for adult livebearers is given a hormonal by oral administration to fulfill the production gap. Only one paper stated that producing of livebearers in platy coral increased the production by using PGF2 $\alpha$ and oxytocin on giving birth [8].

The purpose of this study is to evaluate the effect of OODEV by oral administration during oocyte growth in virgin or non-virgin Poeciliidae fish, including guppy Poecilia reticulata, swordtails Xiphophorus sp., and black molly Poecilia sphenops.

\section{Material and method}

\subsection{Maintenance fish}

Adult wild type of guppy, swordtails, and black molly was purchased from the local pet shop. Then the offspring were maintained until ready to spawn according to Standard maintenance following the Guide for Care and Use of Laboratory Animals published by the US National Institutes of Health.

\subsection{Design experiments}

The experiments were conducted during dry seasons around April to June 2018 at Kolam Percobaan Babakan, Faculty of Fisheries and Marine Science, IPB University. Adult wild guppy Poecilia reticulata, swordtails Xiphophorus sp., and black molly Poecilia sphenops were purchased from the local pet shop. The experiments were conducted in two steps. The first experiments used virgin fish that resulted from the first spawning. Then the parent used for the second experiment, rematuration experiment. The OODEV at $0.5 \mathrm{mg} / \mathrm{kg}$ was mixed with commercial fed by using F-999, PT Central Proteina Prima. OODEV and commercial feed were made freshly before being given to the fish. The virgin fish were fed-treated OODEV for 14 days, then evaluated for 30 days. Then a second experiment used the same 
fish. Emptying gonad was performed for 14 days, and sectioned one fish for represent the status of the gonad. Then fish was fed for 14 days and evaluated for 45 days.

\subsection{Parameters}

Reproductive performance was evaluated from 6 adult female fish in both experiments 1 and 2 , including pregnancy index, frequency birth, and survival larvae for 15 days after birth (dab). The pregnancy index was evaluated from 6 adult fish for both experiments 30 and 45 days after treatment. The frequencies of giving birth were evaluated for 45 days by counting every 3-6 days after birth to avoid larvae stress. To assess whether the yolk sac formation was not a problem during vitellogenesis, the survival rate (SR) was observed using 30 larvae each treatment for 15 dab.

\section{Results}

This study is preliminary research for applying OODEV in livebearer, which has the potential to develop in ornamental fishes in Indonesia. In this research, we tested the effect of OODEV in virgin fishes, meaning the adult female of guppy, swordtails, and black molly are the first time spawning in their life. The fish treated-OODEV showed gestation at 7-9 days after treatment pregnancy index $67-83 \%$ compared to the control (Fig. 1.).

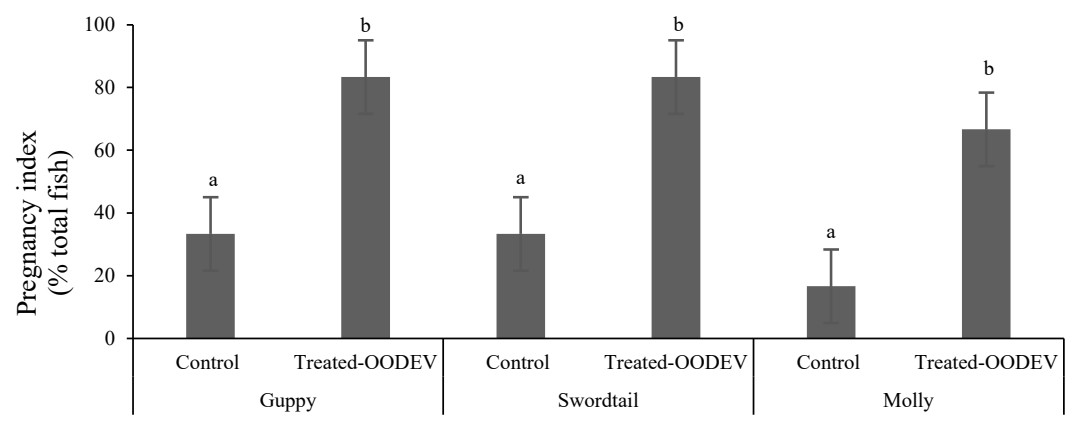

Fig. 1. Pregnancy index of female virgin fish for 14 days observation.

These data indicated that OODEV can accelerate the oocyte growth in livebearer compared to the control. Furthermore, the SR of larvae in this experiment was not affected by OODEV treatment, suggesting that OODEV has an action on the pituitary and gonad, but not the liver (Fig. 2.).

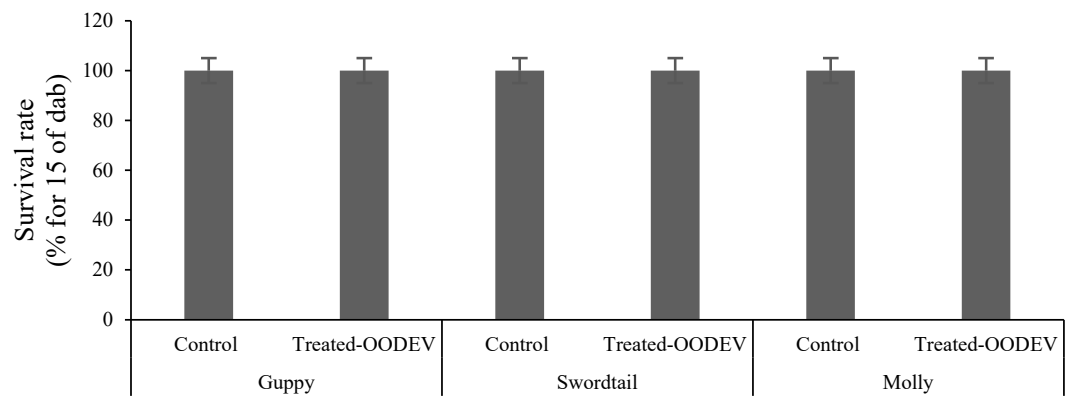

Fig. 2. Survival rate of female virgin fish for 15 days. 
The livebearer, including guppy, swordtail, and black molly, showed acceleration around 4-9 days compared to the control in experiment 2, which used non-virgin of those livebearer fish. Within the 10-13 days after fed-OODEV treatment, fish occurred gestation by signing white spot in the genital pore in all females (Fig. 3.).

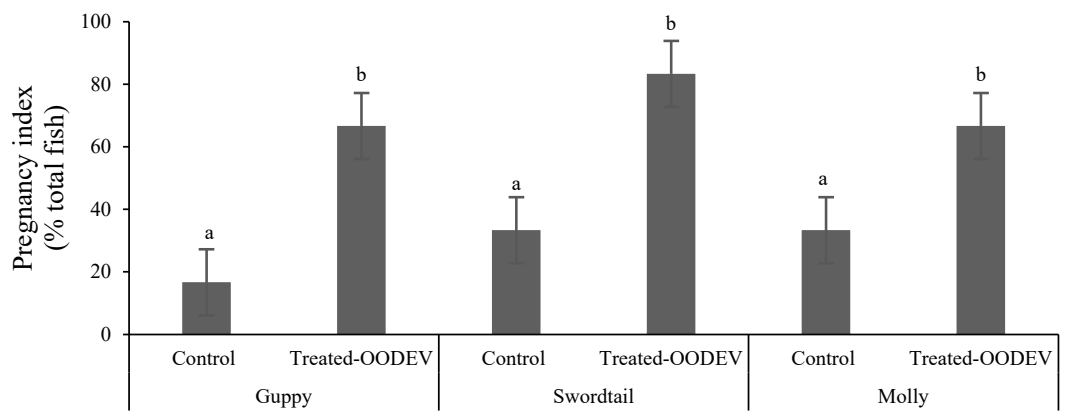

Fig. 3. Pregnancy index of non-virgin fish female for 14 days observation.

In a second experiment, the female fish were used non-virgin. Again, the evidence showed the birth frequency increased compared to the control group (Fig. 4.), suggesting the genital pore has more elasticity than the control, which may be correlated with oxytocin production.

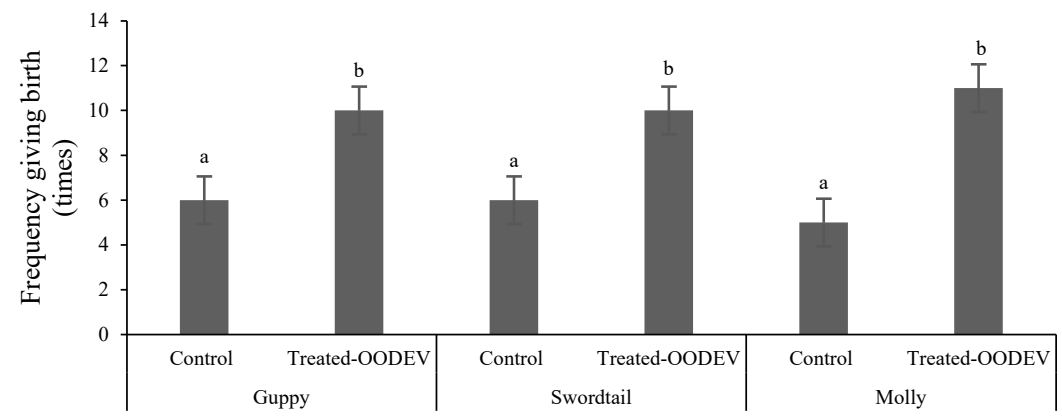

Fig. 4. Frequency adult females take birth for 45 days.

In comparison to the control group, the fish fed-OODEV treatment generated more larvae (Table 1). Indeed, the SR larvae at 15 dab showed similar to the control, suggesting the vitellogenin production occurred with normal conditions during oocyte growth (Fig. 5.).

Table 1. Frequency adult females take birth for 45 days.

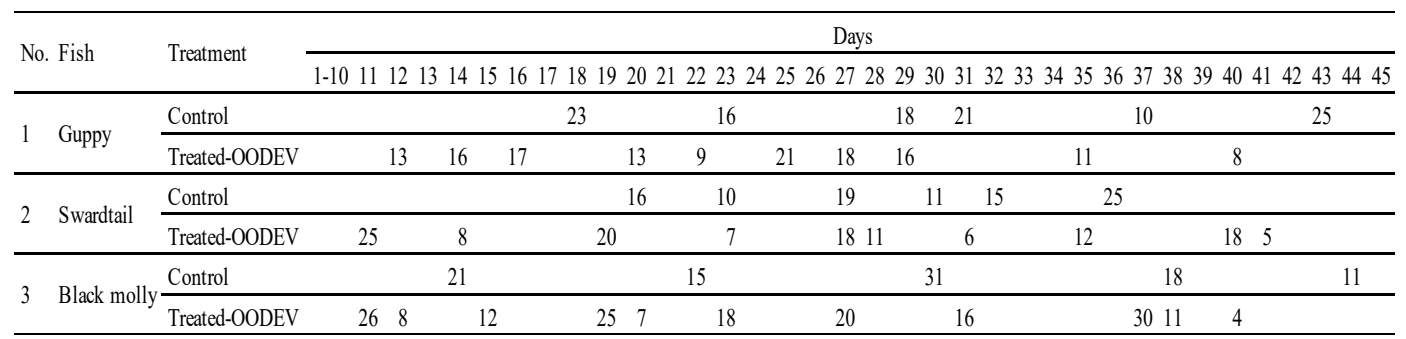




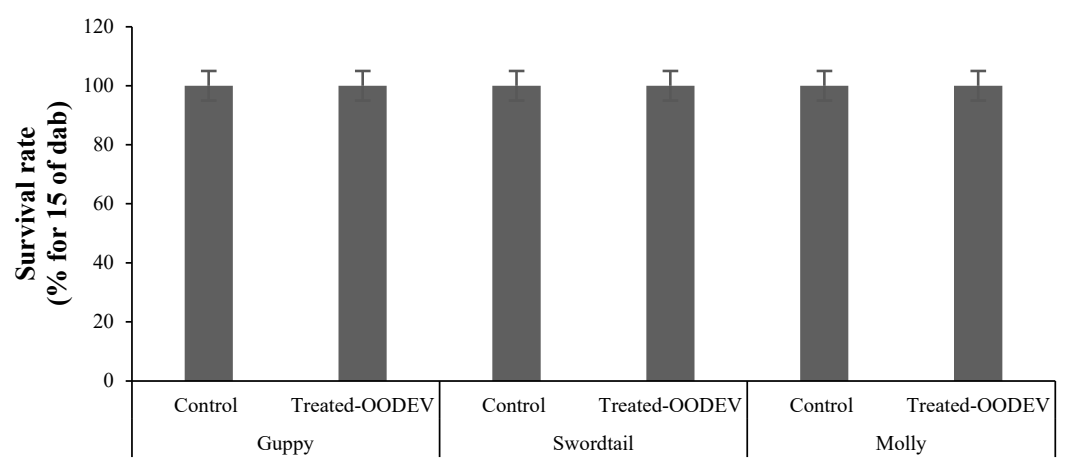

Fig. 5. The survival rate of non-virgin fish females for 15 days.

\section{Discussion}

The result demonstrated that the OODEV could be transferred using oral administration and increased frequencies spawning in the guppy, swordtails, and black molly. The utilization of OODEV in livebearers in this study was preliminary for viviparous fish. The effect of OODEV on oocyte growth was done in several teleosts or invertebrates such as striped catfish, Asian catfish, common carp, tilapia, platydoras, eel, clownfish, Tor soro, snakehead fish, synodontis, and shrimp (Unpublished data). The normal physiology was occurred by stimulating from an external factor, including temperature, light-dark period, $\mathrm{pH}$, and hardness. Those external factors may contribute during vitellogenesis and maturation, though the internal factor such as secretion hormone from the BPG axis [4].

It has been known but not profoundly demonstrated regarding PMSG and domperidone in animals during oocyte growth. The effect of PMSG showed that it could increase estrogen production, indicating that the vitellogenesis occurred in the medaka and may be related to the FSH secreted from the pituitary $[9,10]$. Our experiment 1 showed the fish treatedOODEV in guppy, swordtail, and black molly gestated, which signed a white spot in the genital pore. Afterward, the larvae come out of the ovary. Further research related to anatomy and morphology will be interesting for period active sperm kept in the ovary. In addition, the larvae were evaluated to understand the hormonal manipulation was no effect on the yolk sac during vitellogenesis in the guppy, swordtails, and black molly. Indeed, the yolk sac absorption has many factors, such as temperature or $\mathrm{pH}$.

The temperature and $\mathrm{pH}$ were the same conditions in our case, so we judged that the hormonal manipulation on vitellogenesis was done correctly. Dopamine is known to secrete from dopaminergic neurons and has several physiological activities. It is well documented that dopamine is a neurotransmitter and plays in the BPG axis by inhibiting GnRH, which is controlled by estrogen [7,11]. Therefore, the combination of PMSG and AD in vitellogenesis may increase estrogen production in the brain and stimulate the pituitary-secreted FSH. This process is a response to GnRH from the brain, then induces the ovary to secrete estrogen. The circulating estrogen is brought to the liver cells through blood vessels and binds to the estrogen receptor (ER) [12]. However, estrogen and ER are not the only factors in vitellogenin production. It is stated that female hagfish indicated from hepatic vitellogenin mRNA showed similar significant levels associated with hepatic ER mRNA levels or serum estradiol $\left(E_{2}\right)$ levels [13]. In addition, the presence of estrogen responsive element (ERE) in the promoter region of vitellogenin genes indicated that transcription of vitellogenin might 
control estrogen production in the brain in zebrafish [14]. Further investigation is needed related to transcriptomic analysis in livebearer.

In the second experiment, we tested the same fish for rematuration process. Since we lack the information on period-kept active sperm in micro pockets in the ovary, we expected atresia, the process by which ovarian follicles are degraded with resorption of yolk for recycling so that the gonad will empty by the adult of livebearer. The adult fish treatedOODEV showed a $63-86 \%$ pregnancy index in the guppy, swordtails, and black molly. Due to the body shape of black molly is shorter than guppy and swordtail affected that the percentage of pregnancy was increased. Investigation of the relationship between body shape and period maturation would be a future research interest. Frequencies of birth in adults also showed increased in all livebearer species. These data indicated ovary occurs oocyte growth in adult fish as we know that the atresia is the first step in oocyte development, including oocyte growth and maturation [16]. The proliferation-differentiation-growing-maturation is a step-in oocyte development. In addition, the larvae were not significantly affecting survival for 15 days and indicating no abnormality on forming the vitellogenin during oogenesis in livebearer. We believed that estrogen production might contribute to forming vitellogenin in the liver as a sign of oogenesis in the gonad of livebearer. In line with [9], a fish-treated PMSG increased the aromatase activity in medaka's blood, indicating that PMSG induced estrogen production for forming vitellogenin in the liver. The possible mechanism of molecular level that PMSG induced transcription and translation of vitellogenin dependently. Further investigation is required for possible mechanisms in livebearer for sustainable production.

\section{Conclusion}

In conclusion, our study concluded that OODEV could be transferred to livebearer by oral administration. The OODEV can increase the production of livebearer by rising frequencies spawning a 3-fold increase in out of season. This study can be a reference for viviparous species.

\section{References}

1. A.W. Bell, R.A. Ehrhardt, Nutr. Res. Rev. 15, 211-230 (2002).

2. M.C. Uribe, H.J. Grier, S.A. Avila-Zúñiga, A. García-Alarcón, J. Morphol. 279, 13361345 (2018).

3. H. Kobayashi, T. Iwamatsu. Zoolog. Sci. 19, 545-555 (2002).

4. Y. Nagahama, M. Yamashita, Dev. Growth Differ. 50, S195-S219 (2008).

5. Y. Zohar, C.C. Mylonas, Aquac. 197, 99-136 (2001).

6. C.C. Mylonas, A. Fostier, S. Zanuy, Gen. Comp. Endocrinol. 165, 516-534 (2010).

7. S. Dufour, M.E. Sebert, F.A. Weltzien, K. Rousseau, C. Pasqualini, J. Fish Biol. 76, 129-160 (2010).

8. F. Maulana, M. Zairin Jr., A. Alimuddin, M. Abadi, A.N. Fitrih, J. Akuakultur Indones. 19, 181-189 (2020).

9. Y. Nagahama, A. Matsuhisa, T. Iwamatsu, N. Sakai, S. Fukada, J. Exp. Zool. 259, 53$58(1991)$

10. Nagahama Y., Endocrine regulation of gametogenesis in fish. Int. J. Dev. Biol. 38, 217-229 (1994).

11. Y. Zohar, J.A. Muñoz-Cueto, A. Elizur, O. Kah, Gen. Comp. Endocrinol. 165, 438455 (2010).

12. N. ER, H. HR, Gen. Comp. Endocrinol. 192, 15-24 (2013) 
13. B.J. Reading, L.K. Andersen, Y.W. Ryu, Y. Mushirobira, T. Todo, N. Hiramatsu, Fishes 3, 45 (2018).

14. O. Yilmaz, A. Patinote, T. Nguyen, E. Com, C. Pineau, J. Bobe, Mol. Reprod. Dev. 86, 1168-1188 (2019).

15. M. Alix, O.S. Kjesbu, K.C. Anderson, J. Fish Biol. 97, 607-632 (2020). 